\title{
COVID-19: Salient Aspects of Coronavirus Infection, Vaccines and Vaccination Testing and their Implications
}

\author{
Pradeep K. Pasricha ${ }^{1}$ and Arun K. Upadhayaya ${ }^{2}$ \\ CSIR-National Physical Laboratory \\ New Delhi, India
}

${ }^{1}$ Retired
${ }^{2}$ Environmental Sciences and Biomedical Metrology Division

\begin{abstract}
In the present study, three basic aspects related to COVID-19 are presented.

(a) The occurrence of coronavirus infection is analyzed statistically as number of coronaviruses infected alveolar cells compared to normal alveolar cells in human lungs. The mole concept is used to estimate the number of normal alveolar cells per human lung. The number of coronavirus infections in infected alveolar cells is estimated from the published Lower Respiratory Tract (LRT) load data. The Poisson probability distribution is aptly applied to imply the incubation period of the coronavirus infection to be within day-3 to day-7, with the cumulative probability of $75 \%$. The incubation period within day- 0 to day-10 has a cumulative probability of $98 \%$. It implies a 10-day quarantine to isolate an uninfected individual as a precautionary measure.

(b) Three vaccines to combat COVID-19, which adopt distinct paradigms while preparing them, are analyzed. These are Moderna's mRNA-1273, Oxford-AstraZeneca's ChAdOx1 nCoV19 and Bharat BioTech's COVAXIN. The mole concept is used to estimate the antigen mass density per dose of each of these vaccines as $10 \mathrm{~g} \mathrm{~cm}^{-3}, 0.1 \mathrm{~g} \mathrm{~cm}^{-3}$ and $1 \mathrm{~g} \mathrm{~cm}^{-3}$, respectively. The vaccines are deemed to be compatible to neutralize the infection.

(c) A statistical analysis is performed of the Moderna's mRNA-1273 vaccine efficacy of 94.1\% and Oxford's ChAdOx1 nCoV-19 vaccine efficacy of $62.1 \%$ in terms of groups of volunteers testing negative to vaccine by chance. In the Moderna vaccination testing scenario, since the probability of negative response of vaccine is small, the Poisson probability distribution for $95 \%$ cumulative probability is used to describe the vaccination testing in 300 samples of 47 volunteers each. Thus, $87 \%$ of samples have average group of 3 volunteers testing negative to vaccine. About $6 \%$ of samples have all volunteers testing positive to vaccine. In the Oxford vaccination testing scenario, since the probability of negative response of vaccine is finite, the Gaussian probability distribution for $95 \%$ probability is used to describe the vaccination testing in 75 samples of 120 volunteers each. Thus, $68 \%$ of samples have average group of 45 volunteers testing negative to vaccine. No sample has all volunteers testing positive to vaccine. A vaccine, irrespective of its efficacy being high or low, is necessary for mass immunization.
\end{abstract}




\section{Introduction}

In the present study, three basic aspects related to COVID-19 are presented. These are (i) the statistical analysis of the occurrence of coronavirus infection in human beings, (ii) the analysis of varied vaccines in terms of antigen mass density per dose to neutralize the infection and (iii) the statistical analysis of a vaccine testing procedure in terms of vaccine efficacy. In (i) and (ii), the mole concept, that is, one mole of a substance (chemical, biological, virus, or vaccine) contains Avogadro's number of particles (atoms, molecules, cells, viral particles, or specific antigens) is applied.

(i) A statistical study is made for the appropriate probability distribution to describe the occurrence of coronavirus infection in terms of the number of coronavirus infected alveolar cells vis-à-vis normal alveolar cells in human lung. The number of normal alveolar cells in the human lung is evaluated through the mole concept. The number of infected alveolar cells is estimated from the published Lower Respiratory Tract (LRT, moderate to severe COVID-19 symptoms) viral load data in fourteen patients from Germany and one patient from China. The number of coronavirus infections in infected alveolar cells (per lung volume) is much less than the number of normal alveolar cells (per lung volume). Thus, the Poisson probability distribution may be applied to analyze the probability of occurrence of coronavirus infection in human beings.

(ii) Three vaccines used to combat COVID-19, which adopt distinct paradigms while preparing them, are analyzed. These are Bharat BioTech's COVAXIN, Oxford-AstraZeneca's ChAdOx1 nCoV-19 (AZD1222) and Moderna's mRNA-1273. The vaccine COVAXIN is prepared using live but inactivated SARS-CoV-2 virus. The vaccine AZD1222 is a genetically modified preparation from chimpanzee adeno virus. The vaccine mRNA-1273 is a messenger (m) RNA-based vaccine that carries the SARS CoV-2 virus spike immunogen. The mole concept is used to estimate the antigen mass density per dose of each of these vaccines in order to make a comparative study amongst these vaccines.

(iii) A pertinent parameter to gauge the occurrence and severity of COVID-19 is immunity amongst masses. Consequently, in the so-called Phase 3 of vaccination testing, a large number of volunteers, as finite numbers of volunteers at a number of centers, are tested for vaccine efficacy. In mathematical statistics, a large number of volunteers form a population. The finite numbers of volunteers (at each center) are the samples of the population. A probability distribution of the negative response of volunteers to vaccine in samples is thus evaluated; which enables to determine the vaccine efficacy. A number of vaccines for the COVID-19 pandemic have been developed, with a range of mean vaccine efficacy of $60 \%$ to $95 \%$. The outcome of vaccination testing of Moderna's mRNA-1273 vaccine and Oxford's ChAdOx1 nCoV-19 vaccine in terms of the respective vaccine efficacy are analyzed.

The Moderna's mRNA-1273 vaccine, co-developed by Moderna, Inc., and NIAID (National Institute of Allergy and Infectious Diseases), was administered to 14134 volunteers at 99 centers in Phase 3 in the USA [2, 3, 4]. (The volunteers received two doses of the vaccine.) The volunteers were further grouped into three categories, (a) persons 65 years of age or older, (b) persons younger than 65 years of age who are at risk for severe COVID-19 and (c) persons younger than 65 years of age not at risk for COVID-19. There were subgroups based on gender, 
etcetera. In the statistical analysis of the sample (comprising subsamples), the data on the number of volunteers affected by COVID-19 are treated as random. The mean vaccine efficacy is $94.1 \%$ at preventing symptomatic COVID-19. The 95\% confidence interval (or confidence limits) for the vaccine efficacy is $89.3 \%-96.8 \%$ of the Poisson probability distribution of the sample data (number of affected volunteers, efficacy). These are the range of values of the population mean efficacy, calculated using the sample mean efficacy, with $95 \%$ certainty. In the present study, since the probability of negative response of vaccine is small, the Poisson probability distribution is used to describe the vaccine testing procedure.

The Oxford's ChAdOx1 nCoV-19 vaccine, developed at Oxford University, was administered to 8895 volunteers at 25 centers in Phase 2 and Phase 3 in the UK and Brazil. (The vaccine is popularly known as Oxford-AstraZeneca COVD-19 vaccine AZD1222. The volunteers received two standard doses $(\mathrm{SD}, \mathrm{SD} / \mathrm{SD})$ of the vaccine. The vaccine was also administered to volunteers in South Africa at a number of centers. However, the results of the statistical analysis of the vaccine trials in South Africa have not been reported.) The volunteers were further grouped into three categories, (a) persons 70 years of age or older, (b) persons in the age group 56 - 69 years and (c) persons in the age group 18 - 55 years. There were subgroups based on gender, etcetera. The statistical analysis of the efficacy in the randomized subsamples of volunteers at risk for COVID-19 was affected though a robust Poisson regression model. The mean vaccine efficacy is $62.1 \%$ at preventing symptomatic COVID-19. The $95 \%$ confidence interval (or confidence limits) for the vaccine efficacy is $41.0 \%-75.7 \%$ for the determination of the mean efficacy with $95 \%$ certainty. In the present study, since the probability of negative response of vaccine is finite, the Gaussian probability distribution is used to describe the vaccine testing procedure.

\section{Probability Distribution of Occurrence of Coronavirus Infection}

The Poisson probability distribution is aptly applied to infer the incubation period of the coronavirus infection in human beings.

\subsection{Number of Alveolar Cells in Human Lung}

One may estimate the number of alveolar cells in the human lung as follows. The molecular mass (weight) of biological molecules, such as proteins, in lung tissue cells, is taken as $\mathrm{M}_{\mathrm{w}} \approx 10^{5} \mathrm{Da}$ (dalton, $\mathrm{g} \mathrm{mol}^{-1}$, grams mole ${ }^{-1}$ ) [1, Table 12.1]. The mass density of lung tissue is $\rho$ $\approx 0.3 \mathrm{~g} \mathrm{~cm}^{-3}$ [2]. The average adult lung volume is 1.5 litres $\left(1.5 \times 10^{3} \mathrm{~cm}^{3}\right)$. (The lung volume is the volume of air remaining in lungs after a normal exhalation, called the functional residual capacity.)

Molecular mass $\mathrm{M}_{\mathrm{w}}=10^{5}$ Da implies that 1 mole of lung tissue has a mass of $10^{5} \mathrm{~g}$. $1 \mathrm{~cm}^{3}$ of lung tissue has $\rho / \mathrm{M}_{\mathrm{w}}=0.3 / 10^{5}=3 \times 10^{-6}$ moles.

1 mole contains $6.02 \times 10^{23}$ cells of lung tissue. (The Avogadro constant, $\mathrm{N}_{\mathrm{A}}=6.02 \times 10^{23} \mathrm{~mol}^{-1}$, is the number of atoms, molecules, or cells.)

So, $\mathrm{N}=\mathrm{N}_{\mathrm{A}} \times$ moles $=\mathrm{N}_{\mathrm{A}} \times \rho / \mathrm{M}_{\mathrm{w}}=6.02 \times 10^{23} \times 3 \times 10^{-6}=1.806 \times 10^{18}$ cells occupy a volume of $1 \mathrm{~cm}^{3}$. 
Volume $\mathrm{V}\left(\mathrm{cm}^{3}\right)$ per cell $=1 / \mathrm{N}=\left(1 / \mathrm{N}_{\mathrm{A}}\right) \times \mathrm{M}_{\mathrm{w}} / \rho=1 /\left(1.806 \times 10^{18}\right)=5.537 \times 10^{-19} \mathrm{~cm}^{3}$.

The volume occupied per cell is of the order of $\mathrm{d}^{3}$, where $\mathrm{d}$ is the size parameter of a cell. So, approximately, $\mathrm{d}=\mathrm{V}^{1 / 3}=\left(5.537 \times 10^{-19}\right)^{1 / 3} \approx 0.821 \times 10^{-6} \mathrm{~cm} \approx 8.21 \mathrm{~nm}$ (nanometer, $1 \mathrm{~nm}=$ $10^{-9} \mathrm{~m}$ ). Hence, $1.806 \times 10^{18}$ numbers of cells of size $8.21 \mathrm{~nm}$ occupy a volume of $1 \mathrm{~cm}^{3}$.

The "ready to use" expression of volume $\mathrm{V}\left(\mathrm{nm}^{3}\right)$ of a substance (chemical, biological, virus, or vaccine) in terms of molecular mass $M_{w}$ (Da) and density $\rho\left(\mathrm{g} \mathrm{cm}^{-3}\right)$ is given as [1]

$\mathrm{V}=0.001661 \mathrm{M}_{\mathrm{w}} / \rho$

where 0.001661 is $(1 / 6.02) \times 10^{-2} .6 .02$ is part of the Avogadro number.

Thus, the size parameter $\mathrm{d}(\mathrm{nm})$ is

$$
\mathrm{d}=\left(0.001661 \mathrm{M}_{\mathrm{w}} / \rho\right)^{1 / 3}=0.1184\left(\mathrm{M}_{\mathrm{w}} / \rho\right)^{1 / 3}
$$

Also, the density $\rho\left(\mathrm{g} \mathrm{cm}^{-3}\right)$ is

$$
\rho=0.001661 \mathrm{M}_{\mathrm{w}} / \mathrm{V}=0.001661 \mathrm{M}_{\mathrm{w}} / \mathrm{d}^{3}
$$

The alveoli, minute air sacs in the lungs, provide an enormous surface area for efficient respiration. The tissue cells of alveoli, the alveolar macrophages, are large scavenger cells. They remove foreign bodies such as dust, viruses and bacteria from blood, tissues and organs. In essence, the immune cells, B cells and T cells, types of "small" white blood cells, act on viruses. The T cells "memorize", "identify" and "remember" how to fight the virus infection. The B cells "make" antibodies to neutralize the virus infection before the viruses infect cells. The virus infected "perished" cells are also removed in the process. The size of B cells and T cells, which are in globular shape, is: $\mathrm{d} \approx 8000 \mathrm{~nm}$ [1, Table 12.2]. The alveolar cell size is taken as $\mathrm{d} \approx 8000$ $\mathrm{nm}$. One may estimate the number of alveolar cells of size parameter $8000 \mathrm{~nm}$ in terms of cells of $8.21 \mathrm{~nm}$ in a volume of $1 \mathrm{~cm}^{3}$ as: $1.806 \times 10^{18} \mathrm{x}(8.21)^{3} /(8000)^{3} \approx 2 \times 10^{9}$ cells. The "scaling factor" to transform cells of size parameter $8.21 \mathrm{~nm}$ to cells of $8000 \mathrm{~nm}$ is: $(8.21)^{3} /(8000)^{3} \approx$ $1.08 \times 10^{-9} \approx 10^{-9}$. Since the average lung volume is about $1.5 \times 10^{3} \mathrm{~cm}^{3}$, the estimated number of cells of size parameter $8.21 \mathrm{~nm}$ is: $1.5 \times 10^{3} \times 1.806 \times 10^{18} \approx 2.7 \times 10^{21} \approx 10^{21}$. Also, the number of alveolar cells of size parameter $8000 \mathrm{~nm}$ is: $1.5 \times 10^{3} \times 2 \times 10^{9} \approx 3.0 \times 10^{12} \approx 10^{12}$. The number of alveolar cells is given as $10^{10} \times 10^{ \pm 1}[3]$.

\subsection{Number of Coronavirus Infected Alveolar Cells in Human Lung}

The alveolar cells are the host cells for the replication of coronavirus in the human lung. Normally a human does the breathing process of inhalation and exhalation of air about 16 times a minute, about half a litre $\left(500 \mathrm{ml} \mathrm{or} \mathrm{cm}^{3}\right)$ of air each time called the tidal volume. Not all of that half liter breath reaches the alveoli, the minute air sacs in the lungs. One-third of it shuffles in and out of the windpipe and other passages.

A coronavirus penetrates a cell. It replicates to produce virus particles, the virions. Overwhelmed by the virions, the cell perishes. Then the released virus and virions, collectively the viruses, attack other cells. It is anticipated that one is infected by coronavirus in about ten minutes after close contact with an infected person. It is termed the virion entry into cell [3]. Thus, the virus-infected volume of air that could infect the human lung in ten minutes is about: $10 \times 1 / 3 \times 16 \times 500 \mathrm{~cm}^{3} \approx 2.7 \times 10^{4} \mathrm{~cm}^{3} \approx 10^{4} \mathrm{~cm}^{3}$, which is greater than the lung volume of 1.5 $\mathrm{x} 10^{3} \mathrm{~cm}^{3}$. However, the initial virus dose does not decide the severity of infection, that is, the 
number of infected alveolar cells. It is the activation of an individual's immune system, triggered by the coronavirus, which decides the number of infected alveolar cells. Also, the immune response (immunity) of the human body, in terms of white blood cells (B cells and T-cells) and the presence of antibodies against the virus, varies with individuals. A measure of "healthy" immunity in terms of the amount (mass density, number density) of immune B- and T- cells is unknown. The amount of B- and T-cells is a function of many factors, including age [4]. The number of infected alveolar cells may be estimated in terms of the viral load of an infected individual. The viral load is measured as the quantity of virus "copies" per unit volume (millilitre $\mathrm{e}^{-1}, \mathrm{ml}^{-1}$, or $\mathrm{cm}^{-3}$ ). The viral load data has been reported in fourteen patients from Germany and one patient from China [5]. Two sets of data are given, one each for Upper Respiratory Tract (URT, mild COVID-19 symptoms) and Lower Respiratory Tract (LRT, moderate to severe COVID-19 symptoms, lung infection). (The mild URT symptoms may include fever, without shortness of breath, that is, normal respiratory rate. The moderate LRT symptoms may include breathlessness, high respiratory rate and low blood oxygen levels. The severe LRT symptoms may include breathlessness, higher respiratory rate and lower blood oxygen levels.) In general, the URT virus load is lower than the LRT viral load by two orders of magnitude, that is, by a factor of $100\left(10^{2}\right)$ [5]. The LRT virus load may be used to estimate the infected alveolar cells as follows. The LRT viral load of a "representative" patient\#3 in Germany is about $10^{6}$ copies $\mathrm{ml}^{-1}$ on day-4 and day- 5 of infection. The viral load is about $10^{5}$ copies ml ${ }^{-1}$ from day- 6 to day- 15 of infection. The viral load "exponentially" falls to about $10^{4}$ copies $\mathrm{ml}^{-1}$ from day-16 to day-20. Here, the minimum detectable viral load is $100\left(10^{2}\right)$ copies $\mathrm{ml}^{-1}$. The LRT viral load of the patient in China is about $10^{5}$ copies $\mathrm{ml}^{-1}$ and $10^{7}$ copies ml ${ }^{-1}$ on day- 3 and day-4 - to - day-6 of infection, respectively. Then the viral load "exponentially" falls to about $10^{5}$ copies $\mathrm{ml}^{-1}$ on day- 8 and about $10^{3}$ copies $\mathrm{ml}^{-1}$ on day- 9 - to - day- 11 of infection, respectively. Here, the minimum detectable viral load is $1\left(10^{0}\right)$ copies $\mathrm{ml}^{-1}$. It seems reasonable to assume a viral load of $10^{6}$ copies $\mathrm{ml}^{-1}$ (on day-5) to estimate the number of infected alveolar cells. The average number of virions produced by a single infected cell, the "per cell viral yield", termed Burst size, is $10^{3}$ virions [3]. The coronavirus size parameter is $100 \mathrm{~nm} \mathrm{[3].} \mathrm{Thus,} \mathrm{the}$ number of coronavirus infected alveolar cells is: $10^{6} / 10^{3} \mathrm{ml}^{-1}=10^{3} \mathrm{ml}^{-1}\left(\mathrm{or} \mathrm{cm}^{-3}\right)$. Since the average lung volume is about $1.5 \times 10^{3} \mathrm{~cm}^{3}$, the estimated number of infected alveolar cells in the human lung is: $1.5 \times 10^{3} \times 10^{3} \approx 1.5 \times 10^{6} \approx 10^{6}$.

\subsection{Poisson Probability Distribution of Coronavirus Infection}

The probability of occurrence of coronavirus infection is small. The number of infections in terms of the infected alveolar cells of $10^{3}$ (per lung volume, Section 3 ) is much less than the number of alveolar cells of $10^{9}$ (per lung volume, Section 2). A Poisson distribution function is an analytical form for the probability distribution of such data [6, Chapter 3]. The Poisson probability distribution is applicable when the probability of an event is very small, small, or unknown. For illustration, from Section 3, the LRT viral load per day, over a period of 15 days, are the set of infection events. In the Poisson distribution function, day-5 is roughly the mean of days of infection events, corresponding to the maximum of LRT viral load (on day-5). (The 
model fitted curves in Fig. 2 and Fig. 4B in [5] are bounded essentially only on the left side of the mean, which is considered appropriate for the Poisson probability distribution [6, Chapter 3]). With mean $\mu=5$ day, one may compute the probability of an infection on day- 0 , day- 1 , day$2, \ldots$, day-15 of the occurrence of coronavirus infection. Hence, these probabilities may be multiplied by the viral load of $10^{6}$ copies $\mathrm{ml}^{-1}$ to estimate the viral load and the infected alveolar cells on each day. The maximum LRT viral load is on day-5 of the 15-day infection period, for example. For the Poisson distribution of LRT viral load with day, given by the variable $X$, the mean $\mu$ is considered significant within $\pm \sigma$. One has $X=\mu \pm \sigma$, where $\sigma$ is the standard deviation. Here, $\mu=5$ and $\sigma=\sqrt{ } \mu=\sqrt{5}=2.24 \approx 2$ (day). Thus, $X=5 \pm 2$; or, $3 \leq X \leq 7$. The infection symptoms may appear within day-3 to day-7 of the exposure to infection, mean day-5 $(\mu=5)$, with a $75 \%$ cumulative probability of infections. The range of occurrence of infections 3 $\leq X \leq 7$ is the incubation period [5]. For the mean $\mu$ to be significant within $\pm 2 \sigma, X=\mu \pm 2 \sigma=5$ $+2 \times 2.24 \approx 5 \pm 5(0 \leq X \leq 10)$, the cumulative probability for a Poisson distribution with mean day- $5(\mu=5)$ is $98 \%$. Thus, the cumulative probability of the occurrence of infections is $98 \%$ for the exposure to infection within day-0 to day- 10 .

\section{COVID-19 Vaccines}

A COVID-19 vaccine uses a specific antigen, which stimulates in the human body the development of antibodies through B cells and the response of T cells to neutralize the effect of the antigen. It thereby confers immunity against the COVID-19. Three vaccines are analyzed that adopt distinct paradigms while preparing them. In particular, the antigen mass density per dose of each of these vaccines is calculated. Estimation is made of the mass density of SARS-CoV-2 antibody secreted by B cells. Also, the mass density of B cells, $\mathrm{T}$ cells and antibodies in the human beings is estimated. In an inert solution, the injection dose in the intramuscular/intravenous vaccination is $0.5 \mathrm{ml}$ in each dose for these vaccines.

The Bharat BioTech vaccine, COVAXIN, is prepared using live but inactivated SARS$\mathrm{CoV}-2$ virus (antigen per dose of mass $6 \mu \mathrm{g}$, intramuscular injection dose in a potassium buffer solution of $0.5 \mathrm{ml}$ and vaccine efficacy $81 \%$. COVAXIN is co-developed by the biotechnology company Bharat Biotech and the National Institute of Virology (NIV) of the Indian Council of Medical Research (ICMR) [7].) The molecular mass of SARS-CoV-2 virus, size (d) $100 \mathrm{~nm}$, density $(\rho) \sim 1 \mathrm{~g} \mathrm{~cm}^{-1}$, is $\mathrm{M}_{\mathrm{w}} \sim 10^{9} \mathrm{Da}$ [3]. Thence, the molecular mass of SARS-CoV-2 antigen is taken as $\sim 10^{9} \mathrm{Da}$.

From (3), the density $(\rho)$ of virus $=0.001661 \times 10^{9} / 100^{3} \approx 1.66 \mathrm{~g} \mathrm{~cm}^{-3} \sim 1 \mathrm{~g} \mathrm{~cm}^{-3}$.

1 mole of antigen has a mass of $10^{9} \mathrm{~g}$, and has $6.02 \times 10^{23}$ inactivated viruses.

So, $6 \mu \mathrm{g}$ of antigen has: $6 \times 10^{-6} / 10^{9}=6 \times 10^{-15}$ mole.

Also, $6 \mu$ g has: $6.02 \times 10^{23} \times 6 \times 10^{-15}=3.6 \times 10^{9} \approx 3 \times 10^{9}$ inactivated viruses.

Thus a COVAXIN dose, mass $6 \mu \mathrm{g}$, molecular mass $10^{9} \mathrm{Da}$, has $3 \times 10^{9}$ antigen particles.

The Oxford-AstraZeneca vaccine, ChAdOx1 nCoV-19, AZD1222, is a genetically modified preparation from chimpanzee adenovirus (viral particles per dose of $5 \times 10^{10}$, intravenous injection dose in an inert solution of $0.5 \mathrm{ml}$ and vaccine efficacy $62.1 \%$. AZD1222 
is developed at Oxford University [8].) The molecular mass of Chimpanzee adenovirus, size (d) $\sim 100 \mathrm{~nm}$, is $\mathrm{M}_{\mathrm{w}} \sim 150 \times 10^{6} \mathrm{Da}\left(=1.5 \times 10^{8} \mathrm{Da}\right)$ [9].

A dose of AZD1222 has mass $=1.5 \times 10^{8} \times 5 \times 10^{10} / 6.02 \times 10^{23} \approx 1.2 \times 10^{-5} \mathrm{~g} \approx 10 \mu \mathrm{g}$.

Thus an AZD1222 dose, mass $10 \mu \mathrm{g}$, molecular mass $1.5 \times 10^{8} \mathrm{Da}$, has $5 \times 10^{10}$ antigen particles.

The Moderna vaccine, mRNA-1273, is a messenger (m) RNA-based vaccine that carries the SARS CoV-2 virus spike immunogen (antigen per dose of $100 \mu \mathrm{g}$, intramuscular injection dose in an inert solution of $0.5 \mathrm{ml}$ and vaccine efficacy $94.1 \%$. mRNA-1273 is co-developed by Moderna, Inc., and NIAID (National Institute of Allergy and Infectious Diseases) [10, 11, 12].) The molecular mass of mRNA molecule, a nanowire of radius $(\mathrm{r}) \sim 1 \mathrm{~nm}$ and linear length $(\mathrm{h}) \sim$ $300 \mathrm{~nm}$, volume $\left(\mathrm{V}, \pi \mathrm{r}^{2} \mathrm{~h}\right) \approx 900 \mathrm{~nm}^{3}$, is $\mathrm{M}_{\mathrm{w}} \sim 6 \times 10^{6} \mathrm{Da}$ [13].

So, $100 \mu \mathrm{g}$ of antigen has: $6.02 \times 10^{23} \times 100 \times 10^{-6} / 6 \times 10^{6}=10^{13}$ antigen particles.

Thus an mRNA-1273, mass $100 \mu \mathrm{g}$, molecular mass $6 \times 10^{6} \mathrm{Da}$, has $10^{13}$ antigen particles.

From (3), the density of a COVAXIN antigen dose is: $0.001661 \times 10^{9} / 100^{3} \approx 1.6 \mathrm{~g} \mathrm{~cm}^{-3}$. Also, the density of an AZD1222 antigen dose is: $0.001661 \times 1.5 \times 10^{8} / 100^{3} \approx 0.3 \mathrm{~g} \mathrm{~cm}^{-3}$. Also, the density of an mRNA-1273 antigen dose is: $0.001661 \times 6 \times 10^{6} / 900 \approx 10 \mathrm{~g} \mathrm{~cm}^{-3}$. These are the estimated values of density of an antigen dose of vaccines. It is concluded that the density of an antigen dose is $\sim 10^{0 \pm 1} \mathrm{~g} \mathrm{~cm}^{-3}$. It is instructive to note that the density of normal human red blood (cells) and that of white blood cells are $\sim 1.1 \mathrm{~g} \mathrm{~cm}^{-3}(\mathrm{ml})$. (Normally, the white blood cells density $\left(\approx 1.08 \mathrm{~g} \mathrm{~cm}^{-3}\right)$ is lower than the red blood cell density $\left(\approx 1.1 \mathrm{~g} \mathrm{~cm}^{-3}\right.$.)

The mass density of SARS-CoV-2 antibody, secreted by B cells, may be estimated as follows. The molecular mass of SARS-CoV-2 antibody is $\sim 114$ x $10^{3} \mathrm{Da}$ [14]. The "effective" size of antibody, which is "heavier" Y-shaped molecule, is: $\mathrm{d} \approx 10 \mathrm{~nm}$ [15]. Thus, from (3), the mass density of SARS-CoV-2 antibody is: $0.001661 \times 114 \times 10^{3} / 10^{3} \approx 0.2 \mathrm{~g} \mathrm{~cm}^{-3}$. An estimate of molecular mass of antibodies, secreted by B cells, is: $150 \times 10^{3} \mathrm{Da}$ [15]. Thus, the mass density of antibodies is: $0.001661 \times 150 \times 10^{3} / 10^{3} \approx 0.25 \mathrm{~g} \mathrm{~cm}^{-3}$. The mass density of SARSCoV-2 antibody is comparable to the mass density of antibodies secreted by B cells. It is worthwhile to estimate the mass density of $\mathrm{B}$ and $\mathrm{T}$ cells in white blood cells. The molecular mass of $\mathrm{B}$ and T cells is $\sim 70 \times 10^{3} \mathrm{Da}$ [16]. From (3), with $\mathrm{d} \approx 8000 \mathrm{~nm}$, the mass density of $\mathrm{B}$ and T cells is: $\left.0.001661 \times 70 \times 10^{3} / 8000^{3} \approx 0.2 \times 10^{-9} \mathrm{~g} \mathrm{~cm}^{-3}\left(0.2 \mathrm{ng} \mathrm{cm}^{-3} \text {, nanogram cm}\right)^{-3}\right)$.

\section{COVID-19 Vaccination Testing}

The Poisson probability distribution is applied to analyze Moderna's mRNA-1273 vaccine for $94.1 \%$ vaccine efficacy. The Gaussian probability distribution is applied to analyze Oxford's ChAdOx1 nCoV-19 vaccine for $62.1 \%$ vaccine efficacy. Thereby, the percentage of samples of groups of volunteers testing negative to vaccine is determined for $95 \%$ cumulative probability and $95 \%$ probability of the Moderna's vaccine and Oxford's vaccine, respectively.

\subsection{Statistical Analysis of Moderna's 94.1\% Vaccine Efficacy}

In Phase 3, in Moderna's vaccine testing procedure, there are 14134 volunteers at effectively $297(3 \times 99=297)$ centers at an average of 47.59 volunteers per center $[10,11,12]$. (The number of groups of persons based on the age criterion is 3 at 99 centers.) The present 
study considers the vaccine testing in a population of 14100 volunteers, as $\mathrm{N}=300$ samples of $\mathrm{n}$ $=47$ volunteers each. The vaccine efficacy is $94.1 \%$. Thus, the probability ( $\mathrm{p}$ ) of negative response of vaccine in a sample is $5.9 \% ; \mathrm{p}=0.059$. Also, the average number of volunteers $(\mu)$ in a sample testing negative to vaccine is: $\mu=\mathrm{n} \times \mathrm{p}=47 \times 0.059=2.773(\approx 3)$. Since $\mathrm{p}<<1$ and, therefore, $\mu \ll n$, the Poisson probability distribution aptly describes the vaccine testing procedure. The Poisson distribution function, with mean equal to $2.773(\mu)$ volunteers testing negative to the vaccine, is used to compute the probability of groups of volunteers (say, a group of 3 volunteers) testing negative to the vaccine in a sample. Hence, by chance, any groups of volunteers in 300 samples testing negative to vaccine are determined. These groups of volunteers testing negative for $95 \%$ confidence interval are examined vis-à-vis the average number of volunteers obtained with $89.3 \%$ and $96.8 \%$ vaccine efficacy (95\% confidence interval $89.3 \%$ $96.8 \%)$.

The Poisson probability distribution is appropriate for describing small samples of a large population [6, Chapter 3]. Here, a sample means a finite number of volunteers being tested for vaccine, that is, 47 volunteers. A population refers to a large number of volunteers being tested for vaccine, that is, 14100 volunteers - 300 samples of 47 volunteers each.

The Poisson distribution function is given by

$$
P_{P}(X, \mu)=\mu^{X} e^{-\mu} / X \text { ! }
$$

where $\mathrm{P}_{\mathrm{P}}(\mathrm{X}, \mu)$ is the "discrete" probability of $\mathrm{X}$ number of volunteers in a sample (per sample) testing negative to vaccine (given by the discrete random variable $X$ ), $\mu$ the mean (average) number of volunteers per sample testing negative to vaccine and $\sum_{X} P_{P}(X, \mu)=1$. Here, the probability $(\mathrm{p})$ of a volunteer testing negative to vaccine is $100 \%-94.1 \%=5.9 \%$, that is, $\mathrm{p}=$ 0.059. A sample has $\mathrm{n}=47$ volunteers. So, $\mu=\mathrm{np}=47 \times 0.059=2.773$, which is the average number $(\approx 3)$ of volunteers testing negative to vaccine in a sample (per sample). Given, total number of samples of volunteers $N=300$. The sign "?" means the factorial, $X !=X \times(X-1) \times(X-$ 2) $\mathrm{x}(\mathrm{X}-3) \ldots ; 0 !=1 . \mathrm{N} \times \mathrm{P}_{\mathrm{P}}(\mathrm{X}, \mu)$ is the number of samples having $\mathrm{X}$ number of volunteers testing negative to vaccine, by chance.

The number of samples $(\mathrm{m})$ having no volunteer testing negative is given by $\mathrm{X}=0$,

$$
\begin{aligned}
& \mathrm{P}_{\mathrm{P}}(0,2.773)=2.7730 \times \mathrm{e}^{-2.773} / 0 !=\mathrm{e}^{-2.773}=0.06247 \\
& \mathrm{~m}=\mathrm{N} \times \mathrm{P}_{\mathrm{P}}(0,2.773)=300 \times 0.06247 \approx 18.74
\end{aligned}
$$

Thus, 18.74 samples have no volunteer testing negative, all 47 volunteers testing positive, by chance. The number of volunteers who test positive is: $18.74 \mathrm{x} 47 \approx 881$.

In an analogous manner, one may compute the number of samples $(\mathrm{m})$ of volunteers testing negative for each of variable $X=1, \ldots, X=12$, with a constant $C \equiv P_{P}(0,2.773)=$ 0.06247 .

For example, the number of samples $(\mathrm{m})$ having one volunteer testing negative is given by $\mathrm{X}=1$,

$\mathrm{P}_{\mathrm{P}}(1,2.773)=\mathrm{C} \times 2.7731 / 1 !=0.06247 \times 2.773=0.17323$

$\mathrm{m}=\mathrm{N} \times \mathrm{P}_{\mathrm{P}}(1,2.773)=300 \times 0.17323 \approx 51.97$

Thus, 51.97 samples have 1 volunteer testing negative, 46 volunteers testing positive, by chance. The number of volunteers who test negative is $52(\approx 51.97 \times 1)$. The number of 
volunteers who test positive is $2391(\approx 51.97 \times 46)$. Total number of volunteers is $2443(=52+$ 2391).

The number of samples $(\mathrm{m})$ having two volunteers testing negative is given by $\mathrm{X}=2$,

$\mathrm{P}_{\mathrm{P}}(2,2.773)=\mathrm{C} \times 2.7732 / 2 !=0.06247 \times 7.68953 / 2=0.24018$

$\mathrm{m}=\mathrm{N} \times \mathrm{P}_{\mathrm{P}}(2,2.773)=300 \times 0.24018 \approx 72.05$

Thus, 72.05 samples have 2 volunteers testing negative, 45 volunteers testing positive, by chance. The number of volunteers who test negative is $144(\approx 72.05 \times 2)$. The number of volunteers who test positive is $3242(\approx 72.05 \times 47)$. Total number of volunteers is $3386(=144+$ 3242).

A summary of number of samples of volunteers and number of volunteers therein testing negative (and positive) to vaccine, computed using expression (4), is given in Table 1. The variable $X$ is a measure of number of volunteers in a sample (per sample) testing negative to vaccine. $P_{P}(X, \mu)$ is the corresponding probability. The tabulated numbers of samples of volunteers testing negative $(\mathrm{m})$ are bounded on the left side of the mean of 2-3 $(\mu)$ volunteers testing negative, by chance, and exhibit Poisson probability distribution of variable $\mathrm{X}$. The number of samples of volunteers testing negative is $299.99(\approx 300, \mathrm{~N})$. The average number of volunteers testing negative is 832 , p x $14100=0.059 \times 14100=831.9 \approx 832$, in the population of 14100. Alternately, one has: $\mu$ x $300=2.773$ x $300=831.9 \approx 832$ volunteers testing negative. The average number of volunteers testing negative per sample of 47 (n) volunteers is $2.773(\approx 3$, $\mu)$.

Table 1

Summary of number of samples and volunteers, in 300 samples of 47 volunteers each, testing negative (and positive) for $94.1 \%$ vaccine efficacy, by chance (average number of volunteers testing negative $=832$ )

\begin{tabular}{|c|c|c|c|c|c|c|c|}
\hline & Variable $(\mathrm{X})$ & $\begin{array}{c}\text { Probability } \\
\mathrm{P}_{\mathrm{P}}(\mathrm{X}, 2.773)\end{array}$ & $\begin{array}{c}\text { Cumulative } \\
\text { probability } \\
\sum \mathrm{P}_{\mathrm{P}}(\mathrm{X}, 2.773)\end{array}$ & $\begin{array}{c}\text { Number of } \\
\text { samples } \\
(\mathrm{m})\end{array}$ & $\begin{array}{c}\text { Number of } \\
\text { volunteers } \\
\text { testing } \\
\text { negative }\end{array}$ & $\begin{array}{c}\text { Number of } \\
\text { volunteers } \\
\text { testing } \\
\text { positive }\end{array}$ & $\begin{array}{c}\text { Number of } \\
\text { volunteers }\end{array}$ \\
\hline & 0 & 0.06247 & 0.06247 & 18.74 & 0 & 881 & 881 \\
\hline & 1 & 0.17323 & 0.23570 & 51.97 & 52 & 2391 & 2443 \\
\hline & 2 & 0.24018 & 0.47588 & 72.05 & 144 & 3242 & 3386 \\
\hline & 3 & 0.22201 & 0.69789 & 66.60 & 200 & 2930 & 3130 \\
\hline & 4 & 0.15391 & 0.85180 & 46.17 & 185 & 1985 & 2170 \\
\hline & 5 & 0.08536 & 0.93716 & 25.61 & 128 & 1076 & 1204 \\
\hline & 6 & 0.03945 & 0.97661 & 11.84 & 71 & 485 & 556 \\
\hline & 7 & 0.01563 & 0.99224 & 4.69 & 33 & 188 & 221 \\
\hline & 8 & 0.00542 & 0.99766 & 1.63 & 13 & 64 & 77 \\
\hline
\end{tabular}




\begin{tabular}{|c|c|c|c|c|c|c|c|}
\hline & 10 & 0.00046 & 0.99979 & 0.14 & 1 & 5 & 6 \\
\hline & 11 & 0.00012 & 0.99991 & 0.04 & 0 & 1 & 1 \\
\hline & 12 & 0.00003 & 0.99994 & 0.01 & 0 & 0 & 0 \\
& & $(\approx 1.0)$ & & & & \\
\hline Total & & 0.99994 & & 299.99 & 832 & 13267 & 14099 \\
& & $(\approx 1.0)$ & & $\approx 300)$ & & & $\approx 14100)$ \\
\hline
\end{tabular}

The variability in $X$, in the Poisson probability distribution of variable $X$, is given by the variance $\sigma^{2}=\mu$, where $\sigma$ is the sample standard deviation. Here, standard deviation $\sigma=\mu^{1 / 2}=$ $2.773^{1 / 2} \approx 1.665(\approx 2)$, volunteers testing negative per sample.

From Table 1 , the variable $\mathrm{X}$ for the cumulative probability of 0.93716 (or $93.7 \% \approx$ $95 \%)$ is: $X=5$. It corresponds to $X=\mu+\sigma=2.773+1.665=4.438(\approx 5)$. Since for $X=0$, there are no $(0$, zero) volunteers testing negative to vaccine, one may adopt the variable $\mathrm{X}$ to be significant within $\mu \pm \sigma=2.773 \pm 1.665$; that is, 1.108 to 4.438 (or 1 to 5 ). The variables $X=0$, $1,2,3,4$ and 5 form group of volunteers testing negative to vaccine for $95 \%$ cumulative probability.

In mathematical statistics, the significance of variable $X=5$ is the statement of assurance (certainty) that "not more than" 5 volunteers shall be testing negative to vaccine. The probability that a sample shall "fail to meet" the assurance is

$$
\mathrm{P}_{\mathrm{P}}(\mathrm{X}>5,2.773)=1-\mathrm{P}_{\mathrm{P}}(\mathrm{X} \leq 5,2.773)=1-\sum \mathrm{P}_{\mathrm{P}}(\mathrm{X}, 2.773)
$$

where the summation is from $\mathrm{X}=0$ to $\mathrm{X}=5$. From Table $1, \sum \mathrm{P}_{\mathrm{P}}(\mathrm{X}, 2.773)=0.93716(\approx 0.95)$, implying $\mathrm{P}_{\mathrm{P}}(\mathrm{X}>5,2.773)=0.06284(\approx 5 \%)$, for $95 \%$ cumulative probability.

From Table 1, the number of samples with no volunteer testing negative, all volunteers testing positive, is 18.74 , by chance. The corresponding percentage of samples is: $(18.74 / 300) \mathrm{x}$ $100 \approx 6 \%$.

Also, from Table 1, the number of samples with volunteers testing negative, corresponding to variable $X=1,2,3,4$ and 5 within $\pm \sigma$, is $262.4(\approx 262)$. It is about $88 \%$ of total 300 samples. Alternately, from Table 1, the corresponding number of volunteers testing negative is 709 . It is about $85 \%$ of average 832 volunteers testing negative. Thus, on average, $87 \%$ of samples have average number of $2.773(\approx 3, \mu)$ volunteers testing negative, by chance. It implies that, on average, $724(=832 \times 0.87)$ volunteers test negative in groups of 3 volunteers in 300 samples, by chance.

Also, from Table 1, the number of samples with volunteers testing negative, corresponding to variable $\mathrm{X}=6,7$ and 8 , is 18.16 . It is about $6 \%$ of the total 300 samples. Alternately, from Table 1, the corresponding number of volunteers testing negative is 117 . It is about $14 \%$ of the average 832 volunteers testing negative. Thus, on average, $10 \%$ of samples have $6-7-8$ volunteers testing negative, by chance. It implies that, on average, $83 \approx 832 \mathrm{x}$ $0.10)$ volunteers test negative in groups of 7 in 300 samples, by chance.

A summary of groups of volunteers, average number of samples (\%) and average number of volunteers, testing negative to vaccine in groups of 3 and 7 volunteers, is given in Table 2 . On average, about $87 \%$ and $10 \%$ of samples have volunteers testing negative in groups of 3 
volunteers and 7 volunteers, respectively, by chance. About $0.5 \%$ of samples, corresponding to variable $X=9$ and 10 , have volunteers testing negative to the vaccine as isolated cases, by chance.

Table 2

Summary of groups of volunteers, average number of samples (\%) and average number of volunteers, in 300 samples of 47 volunteers each, testing negative for $94.1 \%$ vaccine efficacy, by chance (average number of volunteers testing negative $=832$ )

\begin{tabular}{|c|c|c|c|c|}
\hline $\begin{array}{c}\text { Number of } \\
\text { volunteers testing } \\
\text { negative }\end{array}$ & $\begin{array}{c}\text { Group of } \\
\text { volunteers } \\
\text { testing negative }\end{array}$ & $\begin{array}{c}\text { Average number } \\
\text { of samples }(\%)\end{array}$ & $\begin{array}{c}\text { Average } \\
\text { number of } \\
\text { volunteers } \\
\text { testing negative }\end{array}$ \\
\hline 0 & 0 & $\begin{array}{c}0 \% \\
(6 \%, \text { all } \\
\text { volunteers } \\
\text { testing positive })\end{array}$ & 0 \\
\hline & $1-5$ & 3 & $87 \%$ & $\begin{array}{c}832 \times 0.87 \\
(\approx 724)\end{array}$ \\
\hline & $6-7-8$ & 7 & $10 \%$ & $\begin{array}{c}832 \times 0.10 \\
(\approx 83)\end{array}$ \\
\hline Total & $9-10$ & - & $0.5 \%$ & $\begin{array}{c}832 \times 0.005 \\
(\approx 4)\end{array}$ \\
\hline & & & $103.5 \%$ & 811 \\
$($ Isolated cases) & & $(\approx 100 \%)$ & $(\approx 832)$ \\
\hline
\end{tabular}

To summarize, on average, about $87 \%$ of samples, with volunteers testing negative within $\mu \pm \sigma \approx 3 \pm 2$, have groups of 3 volunteers testing negative. The standard deviation $(\sigma)$ of the Poisson probability distribution is given by $\sigma=\mu^{1 / 2}=1.665 \approx 2$ volunteers testing negative per sample. About $10 \%$ of samples have groups of 7 volunteers testing negative. By chance, about $0.5 \%$ of samples have volunteers testing negative to the vaccine as isolated cases.

The $95 \%$ confidence interval for the mean $(\mu=2.773)$ of the sample for the Poisson probability distribution of variable $\mathrm{X}$ may be estimated from a $\chi^{2}$ table; for instance, from Reference [17, Table 26.8, page 984]. The 95\% confidence limits for $\mu$ are given by

$$
\chi_{(0.025,2 \mathrm{x}) / 2}^{2} \leq \mu \leq \chi_{(0.975,2(\mathrm{x}+1)) / 2}^{2}
$$

where $\chi_{0.025}^{2}$ and $\chi_{0.975}^{2}$ are the 2.5 th percentile and 97.5 th percentile, respectively, of the $\chi^{2}$ distribution. The discrete variable $\mathrm{x}$ refers to degrees of freedom $(v) ; \mathrm{x}=0$ for a set of observations of a sample. The requisite limits of $\mu=2.773$ are obtained, with $\mathrm{x}=0$, as: $0 \leq \mu \leq$ $3.689(\approx 0 \leq \mu \leq 4)$. (In Reference 17, the Table heading for "Q" for the lower limit of 2.5 th percentile is: $1-0.025=0.975$. The heading for " $Q$ " for the upper limit of 97.5 th percentile is: 1 
$-0.975=0.025 . v=(x+1)$. Thus, $x=0$ is $v=1$, and $x=1$ is $v=2$, etc. $\chi^{2}=2 \mu, \mu=\chi^{2} / 2$. It implies that the range of the population mean $\mu$, with $95 \%$ certainty, is: $0 \leq \mu \leq 4$.

The Moderna's vaccine efficacy, for the confidence interval of $95 \%$ of the Poisson distribution, is in the range $89.3 \%-96.8 \%$ of the population. The limits $89.3 \%$ and $96.8 \%$ mean that the probability (p) of negative response of vaccine is 0.107 and 0.032 , respectively. Thus, the mean $\mu(\mu=47 \mathrm{x} p)$ of the Poisson probability distribution of variable $\mathrm{X}$ per sample is 5.029 and 1.504, respectively. The mean $\mu=1.504$ and $\mu=5.029$ are within $\mu \pm \sigma(=2.773 \pm 1.665)$ of the Poisson probability distribution of the variable X, with $95 \%$ certainty.

\subsection{Statistical Analysis of Oxford's 62.1\% Vaccine Efficacy}

In Phase 3, in Oxford's vaccine testing procedure, there are 4807 volunteers at 19 centers in the UK and 4088 volunteers at 6 centers in Brazil; total of 8895 volunteers at 25 centers [8]. There are 8895 volunteers at effectively $75(3 \times 25=75)$ centers at an average of $118.6(\approx 120)$ volunteers per center. (The number of groups of persons based on the age criterion is 3 at 25 centers.) The present study considers the vaccine testing in a population of 8895 volunteers, as $\mathrm{N}$ $=75$ samples of $\mathrm{n}=118.6$ volunteers each. The vaccine efficacy is $62.1 \%$. Thus, the probability (p) of negative response of vaccine in a sample is $37.9 \% ; p=0.379$. Also, the average number of volunteers $(\mu)$ in a sample testing negative to vaccine is: $\mu=\mathrm{n} \times \mathrm{p}=118.6 \times 0.379=44.949=$ 45.0. The average number of volunteers testing negative is: $\mu$ x $75=45.0 \times 75=3375$ volunteers testing negative. (Alternately, the average number of volunteers testing negative is $3371, \mathrm{p} \mathrm{x}$ $8895=0.379 \times 8895=3371.2(\approx 3375)$, in the population of 8895.) Since $\mathrm{p}$ is finite, the Gaussian (or normal) probability distribution describes the vaccine testing procedure. (The Gaussian distribution function describes the data that is symmetric about the mean [6, Chapter $3]$ ). The Gaussian distribution function, with mean equal to $45(\mu)$ volunteers testing negative to vaccine, is used to compute the probability of groups of volunteers (say, a group of 45 volunteers) testing negative to the vaccine in a sample. Hence, by chance, any groups of volunteers in 75 samples testing negative to vaccine are determined. These groups of volunteers testing negative for 95\% confidence interval are examined vis-à-vis the average number of volunteers obtained with $41.0 \%$ and $75.7 \%$ vaccine efficacy (95\% confidence interval $41.0 \%$ $75.7 \%)$.

The Gaussian distribution function is given by

$$
\mathrm{P}_{\mathrm{G}}(\mathrm{X}, \mu, \sigma)=\left(1 / \sigma(2 \pi)^{1 / 2}\right)^{-1} \exp \left[-1 / 2((\mathrm{x}-\mu) / \sigma)^{2}\right]
$$

where $\mathrm{X}$ is the "continuous" random variable, $\mu$ the mean (average) number of volunteers per sample testing negative to vaccine and $\sigma$ the sample standard deviation of variability in variable $\mathrm{X}$. The variable $\mathrm{X}$, in essence, depicts a range of number of volunteers testing negative to vaccine. The continuous function $\mathrm{P}_{\mathrm{G}}(\mathrm{X}, \mu, \sigma)$ is the probability density such that $\mathrm{dP}_{\mathrm{G}}(\mathrm{X}, \mu, \sigma)$ is the probability of $\mathrm{X}$ (number of volunteers) within an infinitesimal interval $\mathrm{dX}$, around $\mathrm{X}$ : $\mathrm{dP}_{\mathrm{G}}(\mathrm{X}, \mu, \sigma)=\mathrm{P}_{\mathrm{G}}(\mathrm{X}, \mu, \sigma) \mathrm{dX}$. The integral (area) of the distribution function $\mathrm{P}_{\mathrm{G}}(\mathrm{X}, \mu, \sigma)$, $\mathrm{A}_{\mathrm{G}}(\mathrm{X}, \mu, \sigma)$, between the limits $\mu \pm \mathrm{z} \sigma$, is the probability that $\mathrm{X}$ (number of volunteers) deviates from $\mu$ less than $\mathrm{z} \sigma ; \mathrm{z}(=|(\mathrm{X}-\mu)| / \sigma))$ and $\mathrm{A}_{\mathrm{G}}(\mathrm{z}=\infty)=1[6$, Chapter 3$]$. The area under the curve $A_{G}(X, \mu, \sigma)$ versus $z$ is tabulated elsewhere [Reference 6, Table C-2, page 308]. The table of 
$\mathrm{A}_{\mathrm{G}}(\mathrm{X}, \mu, \sigma)$ versus $\mathrm{z}$ is a universal table, for any set of values of $(\mu, \sigma)$. For instance, the probability of $X$ (number of volunteers), around $\mu$, between $\pm \sigma$ is the area $68.3 \%(\approx 68 \%)$. $\mathrm{N} \mathrm{x}$ $\mathrm{A}_{\mathrm{G}}(\mathrm{X}, \mu, \sigma), \mathrm{N}=75$, is the number of samples having a range of $\mathrm{X}$ (number of volunteers) testing negative to vaccine, by chance.

Since the sample size $n=118.6$ is large $(n>100)$, the variability in variable $X$ is given by the variance $\sigma^{2}=n p(1-p)$, where $\sigma$ is the sample standard deviation. $\left(\sigma=(n p q)^{1 / 2}, q=1-p\right.$, for the binomial probability distribution.) Here, standard deviation $\sigma=(118.6 \times 0.379 \times 0.621)^{1 / 2}=$ $5.28=5.3(\approx 5)$, volunteers testing negative per sample.

(a) The mean $\mu$ is significant within $\pm \sigma$. Thus, variable $X$ (number of volunteers) lies within $\mu \pm \sigma=45.0 \pm 5.3$; that is, $39.7(\approx 40.0)$ to $50.3(\approx 50)$. Thus, on average, $68 \%(\approx 68.3 \%)$ of volunteers per sample have 45 - 50 volunteers (average number of 45 volunteers, $\mu$ ) testing negative, by chance. On average, $51(\approx 75 \times 0.683=51.2)$ samples have 45 volunteers negative, by chance. The percentage of samples is: $51.2 / 75=68.3 \%(\approx 68 \%)$. The number of volunteers testing negative is 2295 (= $51 \times 45)$; alternately, $3375 \times 0.68=2295$.

(b) Consider that variable $X$ (number of volunteers) lies within $\pm 1.5 \sigma$, around $\mu, \mu \pm 1.5 \sigma$ $=45.0 \pm 8$; that is, 37 to 53 . The area between $\pm 1.5 \sigma$ is $86.6 \%(\approx 87 \%)$. The difference of area between $\pm 1.5 \sigma$ and $\pm 1 \sigma$ is: $86.6 \%-68.3 \%=18.3 \%(\approx 18 \%)$. Thus, on average, $18 \%$ of volunteers per sample have (i) 37 - 40 volunteers (average number of 39 volunteers) and (ii) 50 53 volunteers (average number of 52 volunteers) testing negative, by chance. (The range of volunteers is obtained by combining with the range of volunteers in (a).) The probability of each group of volunteers in (i) and (ii) is $9 \%$. On average, $7(\approx 75 \times 0.09=6.8)$ samples have (i) 39 volunteers testing negative and (ii) 52 volunteers testing negative, each, by chance. The percentage of samples in each case is $9 \%(6.8 / 75=9 \%)$. The number of volunteers testing negative is $637(=273+364)$; (i) $273(=7 \times 39)$ and (ii) $364(=7 \times 52)$.

(c) Consider that variable $X$ (number of volunteers) lies within $\pm 1.96 \sigma(\approx \pm 2 \sigma)$, around $\mu$, $\mu \pm 1.96 \sigma=45.0 \pm 10.4$; that is, $34.6(\approx 35)$ to $55.4(\approx 55)$. The area between $\pm 1.96 \sigma$ is $95 \%$. The difference of area between $\pm 1.96 \sigma$ and $\pm 1.5 \sigma$ is: $95 \%-86.6 \%=8.4 \%(\approx 8 \%)$. Thus, on average, $8 \%$ of volunteers per sample have (i) 35 - 37 volunteers (average number of 36 volunteers) and (ii) 53 - 55 volunteers (average number of 54 volunteers) testing negative, by chance. (The range of volunteers is obtained by combining with the range of volunteers in (b).) The probability of each group of volunteers in (i) and (ii) is about 4\%. On average, $3(75 \times 0.04=3)$ samples have (i) 36 volunteers testing negative and (ii) 54 volunteers testing negative, each, by chance. The percentage of samples in each case is: $3 / 75=4 \%$. The number of volunteers testing negative is $270(=108+162)$; (i) $108(=3 \times 36)$ and (ii) 162 (= $3 \times 54)$.

A summary of average groups of volunteers, average number of samples (\%) and average number of samples, of volunteers testing negative to vaccine in 75 samples is given in Table 3 . 
Table 3

Summary of average groups of volunteers, average number of samples (\%) and average number of samples, in 75 samples of 120 volunteers each, testing negative for $62.1 \%$ vaccine efficacy, by chance

\begin{tabular}{|c|c|c|c|c|}
\hline & $\begin{array}{c}\text { Range of number } \\
\text { of volunteers } \\
\text { testing negative }\end{array}$ & $\begin{array}{c}\text { Average group of } \\
\text { volunteers } \\
\text { testing negative }\end{array}$ & $\begin{array}{c}\text { Average number } \\
\text { of samples }(\%)\end{array}$ & $\begin{array}{c}\text { Average number } \\
\text { of samples }\end{array}$ \\
\hline & $35-37$ & 36 & $4 \%$ & 3 \\
\hline & $37-40$ & 39 & $9 \%$ & 7 \\
\hline & $40-50$ & 45 & $68 \%$ & 51 \\
\hline & $50-53$ & 52 & $9 \%$ & 3 \\
\hline & $53-55$ & 54 & $4 \%$ & 71 \\
\hline Total & & & $94 \%$ & \\
& & & & \\
\hline
\end{tabular}

The average number of samples for $95 \%$ probability is 71 , which is less than the total number of samples of 75 . The number of volunteers testing negative below 35, and above 55, is described by a random fluctuation; the probability is $0.025(2.5 \%)$.

To summarize, on average, about $68 \%$ of samples, with volunteers testing negative within $\mu \pm \sigma \approx 45 \pm \sigma \approx 45 \pm 5$, have groups of 45 volunteers testing negative. The standard deviation $(\sigma)$ of Gaussian probability distribution is given by $\sigma \approx(\mathrm{npq})^{1 / 2}, \mathrm{q}=1-\mathrm{p} ; \sigma=(118.6 \mathrm{x}$ $0.379 \times 0.621)^{1 / 2}=5.28=5.3(\approx 5)$, volunteers testing negative per sample. About $18 \%$ of samples, with volunteers testing negative within $\{(\mu \pm 1.5 \sigma)-(\mu \pm \sigma)\}$ have groups of 39 volunteers and 52 volunteers testing negative. The percentage of samples in each case is $9 \%$. About $8 \%$ of samples, with volunteers testing negative within $\{(\mu \pm 1.96 \sigma)-(\mu \pm 1.5 \sigma)\}$ have groups of 36 volunteers and 54 volunteers testing negative. (The area between $\mu \pm 1.96 \sigma$ is $95 \%$.) The percentage of samples in each case is $4 \%$.

For the Gaussian probability distribution of variable X, the $95 \%$ confidence limits of the population mean $(\mu)$ based on a single sample, are calculated as:

$$
\left(45-\mathrm{z} .975 \mathrm{X} \sigma / \mathrm{n}^{1 / 2}\right)<\mu<\left(45-\mathrm{z} .025 \mathrm{x} \sigma / \mathrm{n}^{1 / 2}\right)
$$

where the sample standard deviation $\sigma=5.3$ and $n^{1 / 2}=118.6^{1 / 2}=10.89=10.9(\approx 11)$. The 2.5 th percentile and 97.5th percentile limits of the Gaussian probability distribution, in terms of the variable $\mathrm{z}(=|(\mathrm{X}-\mu)| / \sigma))$, are: $\mathrm{z}_{.025}=-1.96$ and $\mathrm{z}_{.975}=1.96$. It implies that the range of population mean $\mu$, with $95 \%$ probability, is: $44<\mu<45$.

The Oxford's vaccine efficacy, for the confidence interval of $95 \%$, is in the range $41.0 \%$ $75.7 \%$ of the population. The limits $41.0 \%$ and $75.7 \%$ mean that the probability (p) of negative response of vaccine is 0.59 and 0.243 , respectively. Thus, the average number of volunteers testing negative per sample $(\mu=188.6 \mathrm{x}$ p) is about 29 and 70 , respectively. These average numbers of volunteers, testing negative to vaccine, are not within 35 to $55(\mu \pm 1.96 \sigma)$ of the Gaussian distribution of the variable X, with $95 \%$ certainty. 


\section{Conclusion}

The three basic aspects of COVID-19 are presented in the present study.

The occurrence of coronavirus infection in human beings is statistically analyzed in terms of the number of coronavirus infected alveolar cells compared to normal alveolar cells in human lungs. The mole concept, that is, one mole of a substance (biological, virus, or vaccine) contains Avogadro's number of particles (cells, viral particles, or specific antigens) is applied. It leads to the number of normal alveolar cells of size parameter $8000 \mathrm{~nm}$ per human lung volume as $10^{9}$. The number of coronavirus infections in terms of infected alveolar cells is estimated from the published Lower Respiratory Tract (LRT) load data in patients from Germany and China. The number of infected alveolar cells of size $100 \mathrm{~nm}$ per human lung volume is $10^{3}$. The number of coronavirus infections in infected alveolar cells (per lung volume) is much less than the number of normal alveolar cells (per lung volume). The Poisson probability distribution is aptly used to imply the appearance of symptoms, the incubation period, of coronavirus infection to be within day-3 to day-7of the exposure to infection. The cumulative probability, with mean incubation on day- 5 , is $75 \%$. The cumulative probability, with mean incubation on day-5, for the infection to be between day-0 to day-10 of the exposure to infection is $98 \%$.

Three vaccines to combat COVID-19, which adopt distinct paradigms while preparing them, are analyzed. These are Moderna's mRNA-1273, Oxford-AstraZeneca's ChAdOx1 nCoV19 (AZD1222) and Bharat BioTech's COVAXIN. The vaccine mRNA-1273 is a messenger (m) RNA-based vaccine that carries the SARS CoV-2 virus spike immunogen. The vaccine AZD1222 is a genetically modified preparation from chimpanzee adenovirus. The vaccine COVAXIN is prepared using live but inactivated SARS-CoV-2 virus. The mole concept leads to the antigen mass density per dose of each of these vaccines as $10 \mathrm{~g} \mathrm{~cm}^{-3}, 0.1 \mathrm{~g} \mathrm{~cm}^{-3}$ and $1 \mathrm{~g} \mathrm{~cm}^{-3}$, respectively. Thus, the estimated mass density of an antigen dose is $\sim 10^{0 \pm 1} \mathrm{~g} \mathrm{~cm}^{-3}$. The vaccines are deemed to be compatible to neutralize the infection.

A statistical analysis is performed of the Moderna's vaccine efficacy of $94.1 \%$ and Oxford's vaccine efficacy of $62.1 \%$ in terms of groups of volunteers testing negative to the vaccine, by chance, for $95 \%$ certainty. The Moderna's mRNA-1273 vaccination testing scenario comprises 300 samples of 47 volunteers each. The Moderna's $94.1 \%$ vaccine efficacy, probability $\mathrm{p}=0.059(5.9 \%)$, implies that on average 3 volunteers per sample have a negative response to vaccine, by chance. Since the probability of negative response of vaccine is small, the Poisson probability distribution for $95 \%$ cumulative probability is used to describe the vaccination testing. Thus, $87 \%$ of samples (i.e., 261 samples) have groups of 3 volunteers testing negative to the vaccine. Also, $10 \%$ of samples (i.e., 30 samples) have groups of 7 volunteers testing negative to vaccine. About $0.5 \%$ of samples (i.e., 2 samples) have groups of $9-10$ volunteers testing negative to vaccine, with a small probability. About $6 \%$ of samples (i.e., 18 samples) have all volunteers testing positive to vaccine. (The total number of samples is 311, which is roughly 300 samples.) The Oxford's ChAdOx $1 \mathrm{nCoV}-19$ vaccination testing scenario comprises 75 samples of 120 volunteers each. The Oxford's $62.1 \%$ vaccine efficacy, probability $\mathrm{p}=0.379(37.9 \%)$, implies that on average 45 volunteers per sample have a negative response to vaccine, by chance. Since the probability of negative response of vaccine is finite, the Gaussian 
(or normal) probability distribution for $95 \%$ probability is used to describe the vaccine testing. Thus, $68 \%$ of samples (i.e., 51 samples) have groups of 45 volunteers testing negative to vaccine. Also, $9 \%$ of samples (i.e., 7 samples) of groups of 39 volunteers, and another $9 \%$ of samples (i.e., 7 samples) of groups of 52 volunteers, test negative to the vaccine. Additionally, $4 \%$ of samples (i.e., 3 samples) of groups of 36 volunteers, and another $4 \%$ samples (i.e., 3 samples) of groups of 54 volunteers, test negative to vaccine. No sample has all volunteers testing positive to vaccine. (The total number of samples is 71 . In addition, about four samples may have a random fluctuation of groups of volunteers below 35 volunteers or above 55 volunteers, with a low probability.) A generalized vaccination testing procedure is one in which both sample size of volunteers being vaccinated and number of samples is 100 , each, in order to evaluate vaccine efficacy. It makes the total number of samples 200, including the placebo group of volunteers.

The statistical study of the occurrence of coronavirus infection implies that the 10-day quarantine be adopted to isolate an individual as a precautionary measure. The compatibility study of the vaccines implies that the mixed doses of the vaccines may be used to neutralize the infection. The statistical analysis of the testing procedure provides, in general, the probabilistic distribution of samples of volunteers testing negative to the vaccine. A vaccine, irrespective of its efficacy being high or low, is necessary for mass immunization to slow and prevent the spread of infection the COVID-19 pandemic.

\section{Acknowledgements}

The authors gratefully acknowledge Dr. B. Madhava Reddy and Dr. P.N. Vijaykumar, formerly of the National Physical Laboratory, for their helpful comments.

\section{References}

[1] Poole, Jr., C. P. and Owens, F. J., Introduction to Nanotechnology, Wiley India, 2007, Chapters 11 and 12, 388 pp.

[2] Bushong, S. C., Workbook for Radiologic Science for Technologists: Physics, Biology and Protection, Elsevier Science, Netherlands, 2017, Chapter 10, 288pp.

[3] Bar-On, Y. M., Flamholz, A., Phillips, R. and Milo, R., SARS-CoV-2 (COVID-19) by the numbers, eLife, vol. 9, e57309, 9 April 2020.

[4] Sahoo, S., Hari, K., Jhunjhunwala and Jolly, M. K., preprint, 16 May 2020.

https://www.biorxiv.org/content/10.1101/2020.05.16.097238v1

[5] Wang, S., Pan, Y., Wang, Q., Miao, H., Brown, A. N. and Rong, L., Modeling the viral dynamics of SARS-CoV-2 infection, Math Biosci., vol. 328: 108438, 1 October 2020.

https: //doi.org/10.1016/j.mbs.2020.108438 (6 August 2020)

[6] Bevington, P. R., Data Reduction and Error Analysis for the Physical Sciences, McGraw-Hill Book Company, New York, 1969, 336 pp.

[7] Bharat Biotech, COVAXIN, SARS-CoV-2 Vaccine.(Bharat Biotech International Limited, Genome Valley, Turkapally, Shamirpet, Hyderabad, Telangana, India.)

https://www.bharatbiotech.com/images/covaxin/covaxin-fact-sheet.pdf 
[8] Merryn Voysey, et al., "Safety and efficacy of the ChAdOx1 nCoV-19 vaccine (AZD1222) against SARS-CoV-2: an interim analysis of four randomized controlled trials in Brazil, South Africa, and the UK", The Lancet, Volume 397, Issue 10269, 09 January 2021, p. 99 - 111. https://www.thelancet.com/pdfs/journals/lancet/PIIS0140-6736(20)32661-1.pdf https://www.thelancet.com/journals/lancet/article/PIIS0140-6736(20)32661-1/fulltext [9] Google: "molecular mass of chimpanzee adenovirus".

[10] The NIH Record, "NIH-Moderna Covid-19 Vaccine Shows Promising Results", Vol. LXXII, No. 25, USA, December 11, 2020.

https://nihrecord.nih.gov/2020/12/11/nih-moderna-covid-19-vaccine-shows-promising-interimresults

[11] Lindsey R. Baden, et al., "Efficacy and Safety of the mRNA-1273 SARS-CoV-2 Vaccine", New England Journal of Medicine, DOI: 10.1056/NEJMoa2035389, 30 December 2020. https://www.nejm.org/doi/full/10.1056/NEJMoa2035389

[12] Sara E. Oliver, et al., "The Advisory Committee on Immunization Practices' Interim Recommendation for Use of Moderna COVID-19 Vaccine - United States, December 2020", CDC (Centers for Disease Control and Prevention), Morbidity and Mortality Weekly Report (MMWR), Weekly / January 1, 2021 / 69(5152); 1653-1656.

https://www.cdc.gov/mmwr/volumes/69/wr/mm695152e1.htm?s_cid=mm695152e1_w

[13] Google: "molecular mass and volume of mRNA molecule".

[14] Google: "molecular mass of SARS-CoV-2 protein".

[15] Google: "molecular mass and size of antibodies".

[16] Google: "molecular mass of B and T cells".

[17] Abramowitz, M. and Stegun, I. A. (Editors), Handbook of Mathematical Functions with Formulas, Graphs, and Mathematical Tables, 9th Printing, Dover Publications, Inc., New York, 1972, 1045 pp. 